Research Article

\title{
Polycystic Ovarian Syndrome: Associated Diseases and Advancements Through Time
}

\author{
Bharat Kwatra*, Mugdha Barik, Chelsea Rumao \\ Invenzion Labs Inc, KIIT School of Biotechnology- Bhubaneswar, St. Xavier's College (Autonomous)- Mumbai, KIIT School of \\ Biotechnology, Bhubaneswar, India. \\ *Corresponding author's E-mail: bkwatra999@gmail.com
}

Received: 12-04-2021; Revised: 26-05-2021; Accepted: 04-06-2021; Published on: 15-06-2021.

\section{ABSTRACT}

This article is an examination of Polycystic Ovarian Syndrome. The scientific development and subsequent research on various aspects of Polycystic Ovarian Disease and Syndrome continue to influence researchers all over the globe today. This article examines the research done and published by researchers and scientists considering current trends and data in scientific queries and demonstrates further aspects of Polycistronic Ovary Syndrome. Additionally, this article explores options for its Diagnosis and Treatment while throwing light on its history.

Keywords: Hyperprolactinemia, Hyperandrogenism, Anovulation, Hirsutism, Oligomenorrhea, Anti Mullerian Hormone, Ovarian Steroidogenesis.

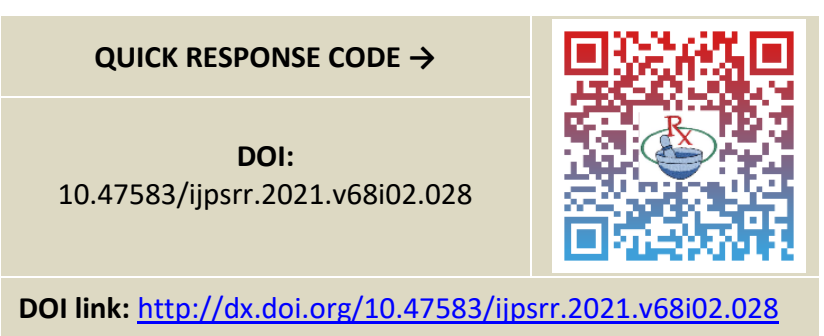

\section{INTRODUCTION}

olycystic ovary syndrome (PCOS) is one of the most common endocrinopathies of women, affecting between 7 and $10 \%$ of all reproductive-aged women and a global prevalence of $5-15 \%$, with significant associated morbidities. Its pathophysiology is not completely understood and its etiology remains an enigma. It is the main cause of infertility in women of reproductive age and can cause other metabolic-related disorders. Nevertheless, there is no single accepted definition for this syndrome. Depending on the defining body, we can have different criteria to define PCOS. There are three major definitions namely (1) NIH definition (2) Rotterdam definition (3) AE-PCOS society definition [Table 1]. All the definitions include 3 main categories: hyperandrogenism, polycystic ovaries, and oligomenorrhea. Based on the NIH diagnostic criteria, there is a prevalence of PCOS of between $6 \%$ and $9 \%$ documented across the United States, the United Kingdom, Spain, Greece, Australia, and Mexico, there are no racial or ethnic influences on the prevalence of PCOS. ${ }^{10,24}$ PCOS is characterized by polycystic ovaries, chronic anovulation (oligo-ovulation or anovulation), and hyperandrogenism leading to the symptoms of irregular menstrual cycles, hirsutism, and acne. This can induce an increased risk of Cardiovascular diseases, Type-2 Diabetes, dyslipidemia, endometrial cancer, and other psychiatric disorders like anxiety. Other subclinical manifestations include sleep apnea, depression, nonalcoholic fatty liver disease, cardiovascular diseases, etc.

Table 1: Definitions of Polycystic Ovary Syndrome

\begin{tabular}{|c|c|c|c|}
\hline & Hyperandrogenism & Oligomenorrhea / Oligoovulation & Polycystic Ovaries \\
\hline NIH & Yes & Yes & Optional \\
\hline Rotterdam & Any 2 out of 3 & \\
\hline AE-PCOS & Yes & Any 1 out of 2 & \\
\hline
\end{tabular}

The diagnosis of PCOS needs to be timely and as precise as possible to prevent complications. Due to the inconsistency in the definition of PCOS and the difference in the patient's clinical symptoms across the globe, a set of international guidelines need to be followed during diagnosis. The contemporarily available diagnostic tests are centered around the features of hyperandrogenism and/or hyperandrogenemia, oligo-ovulation, and polycystic ovarian morphology. The inconsistencies in the tests are mainly due to the influence of environmental and genetic factors on the progression of the disease. AntiMullerian Hormone(AMH), also named as Mullerian inhibiting substance (MIS) is responsible for 2 important stages of folliculogenesis: (1) recruitment of follicles from 
dormant primordial follicle pool (2) cyclic recruitment in which FSH-specific antral follicles are selected for growth. Thus, we can infer that $\mathrm{AMH}$ levels are intricately related to the presence of polycystic ovaries and thus can be used as biomarkers for the diagnosis. Furthermore, a case study was conducted on 41 individuals with newly diagnosed PCOS and 54 healthy women. The study was to predict a connection between patients suffering from PCOS and their unusual levels of calprotectin so that it could be used as a potential biomarker. It has been studied that insulin and insulin resistance play a crucial role in the progression of the disease. Thus, insulin can also be considered to be a very potent biomarker. Lastly, studies have shown that certain types of mi-RNA levels are elevated in PCOS patients as compared to normal healthy women due to which mi-RNA 93 has been proposed as a novel noninvasive biomarker for PCOS diagnosis. Although several non-invasive and novel biomarkers are under tests and scrutiny for being potential solutions to the aforementioned problems, none of them have been approved so far.

Diagnosis and Treatment should be done early to ensure good adulthood health in general. Treatments for women with PCOS include modification of diet, exercise, metformin, and use of Oral Contraceptive Pills. ${ }^{4,5}$ Insulin sensitizers like Thiazolidinediones rosiglitazone and pioglitazone are also used as treatment options. ${ }^{4,5,7}$ Certain herbal medications have also proved to be beneficial for women with PCOS. ${ }^{13}$ The treatment is guided by the symptoms presented by the patient and does not consist of one tone, a combination therapy is used. Long-term follow-up is needed to determine the effectiveness of all the approaches.

Several studies have examined the genetic basis of polycystic ovary syndrome with varying results, probably caused by the different criteria used to define the syndrome. Insulin potentiated Luteinizing hormonedependent (LH-dependent) stimulation of testosterone production by $\mathrm{T}-\mathrm{I}$ cells and $\mathrm{FSH}$-dependent stimulation of $17 \beta$-oestradiol production by $G$ cells but did not significantly affect progesterone production by $\mathrm{T}-\mathrm{I}$ cells or $\mathrm{G}$ cells in the presence or absence of gonadotropins. ${ }^{1}$ The presence of one minor criterion suggests a tendency for PCOS, the presence of two minor criteria would suggest a mild form of PCOS. Polycystic ovary syndrome is one of the most common endocrinopathies among women. ${ }^{18}$ Nevertheless, there is no single accepted definition for this syndrome, its pathophysiology is not completely understood and its etiology remains an enigma.

Polycystic ovary syndrome increases the risk of infertility, endometrial cancer, abnormal glucose metabolism, and dyslipidemia. Strategies such as lifestyle modification, hair removal, and combined oral contraceptive therapy, and other pharmacotherapies are reviewed. The proportion of women with involuntary infertility was $17.5 \%$ in the PCOS group compared with $1.3 \%$ in the control group. Studies have found that the excessive consumption of Western- type food and increasing obesity very lowly affect the prevalence of PCOS which is similar across countries with different rates of obesity. ${ }^{10}$ Also, the mortality and morbidity from coronary heart disease did not differ significantly between the women with PCOS and comparison groups; the most recent search was conducted on 15 October 2018. ${ }^{24}$

\section{METHODS}

The study was conducted using four databases: Google Scholars, SAGE, DOAJ, and PubMed. The selection of papers was done based on keywords and themes relevant to this review. Further, the published papers from these databases were arranged in systematic order with respect to the year of publication

\section{RESULTS AND DISCUSSIONS}

\section{History}

Polycystic ovary syndrome (PCOS) appears to be an ancient disorder perhaps dating at least 50,000 years ago, which has persisted in human evolution despite reduced fecundity because of the benefits to affected women such as greater sturdiness and improved energy utilization, a rearing advantage for their children and kin, and a reduction in the risk of perinatal mortality. This raises the possibility that gene variants that are eventually found to be associated with PCOS will be similar across ethnic groups and races. Stein and Leventhal are regarded to have been the first investigators of polycystic ovary syndrome (PCOS); however, in 1721 Vallisneri, an Italian scientist, described a married, infertile woman with shiny ovaries with a white surface, and the size of pigeon eggs.

Shared genetic susceptibility of PCOS among different populations suggests that genetic risk factors were already present in the ancestors of humans. Contemporary human genetic studies inform us that the origin of human ancestors is from Africa. Sharing common susceptibility loci between Chinese and European ancestry suggests that PCOS may have persisted for more than 50,000 years, before the migration of humans out of Africa. Although PCOS is the most common cause of anovulatory infertility, its high prevalence is still a paradox. From an evolutionary perspective, the pathogenic mechanisms underlying PCOS might be candidate factors for the survival advantage of the human being. Former compensatory advantageous factors may become pathogenic mechanisms underlying complex metabolic disease with prolonged life expectancy and transition to a sedentary lifestyle.

Despite a long history of studies on PCOS, its etiology is still unknown and the very definition remains controversial. ${ }^{18}$ In a thematic analysis of the year 1993, the PCOS patients experienced a variety of symptoms including hirsutism, obesity, acne, and infertility. The participants also suffered from other conditions, both physical and psychological, in addition to their PCOS. Four major themes emerged from the data: change (to life plans and changing nature of condition); comorbidities (living with other conditions and 
depression, self-harm, and suicidal ideation); support (healthcare professionals, education, and relationships) and identity (feminine identity and us and them). Metrodin HP was administered S.C. to stimulate multiple follicular developments in women undergoing in-vitro fertilization (IVF) and embryo transfer. The study showed that only very low concentrations of $\mathrm{LH}$ are required in conjunction with FSH for the stimulation of follicular development and ovarian steroidogenesis. Metrodin $\mathrm{Hp}$ was well tolerated locally thus enabling convenient self-administration without compromising safety or efficacy.

The phenotype of PCOS can be subdivided into four different types. Phenotype A and B (hyperandrogenism + ovulatory dysfunction, with $[A]$ and without $[B]$ polycystic ovarian morphology [PCOM], respectively) can be considered to represent the "classic" form of the disorder. Phenotype C is the so-called "ovulatory" PCOS (hyperandrogenism + PCOM only). And phenotype D is often referred to as "non-hyperandrogenic" PCOS (ovulatory dysfunction + PCOM only). The different phenotypes vary in the degree to which they are associated with an increased risk for metabolic dysfunction and reproductive complications. There are several determinants of the epidemiology (prevalence) and presentation (phenotype) of PCOS, including environmental (e.g., socioeconomic, geographic, toxicologic, lifestyle, and dietary) and genetic (e.g., gene variants, epigenetic, and race/ethnicity) factors. Finally, a better understanding of the evolutionary determinants of PCOS has the potential for providing additional insight into those factors determining the etiology, prevalence, and persistence of a disorder that appears to be, superficially at least, an evolutionary paradox.

\section{Defining Factors Of PCOS}

Hyperandrogenism as a Criteria- The biochemical evidence used while considering hyperandrogenism as a criterion is the measurement of androgen levels. The question at hand was which of the androgen hormones to focus on. The hormone which was ultimately shortlisted was testosterone but there were problems with it since testosterone can be in different forms in the body. Moreover, the reference range to be considered for diagnosing PCOS patients could not be set as a universal value due to there being a disparity in the characteristics of the population. Further uncertainties were faced when deciding on the clinical evidence of hyperandrogenism.

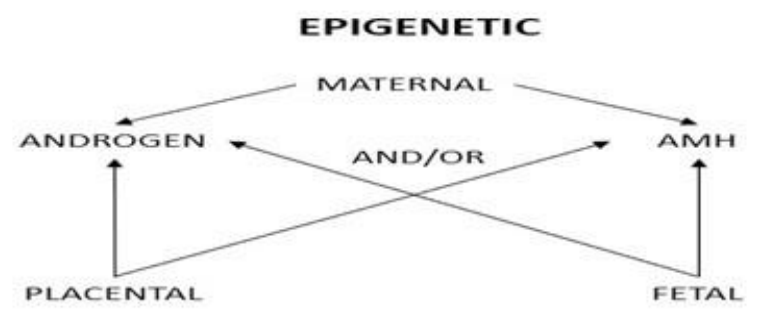

There were discussions about whether to include only hirsutism or also consider the presence of acne and diffuse female alopecia.

Ovulatory Dysfunction as a Criteria- Similar to the problems faced while setting a limit to define hyperandrogenism, there were issues while determining the number of average menstrual counts to consider for a patient to be PCOS positive. "For example, in the studies of Treloar and colleagues, the upper reference limit menstrual interval appears to be 34 days or less. Thus, women who have menstrual intervals of greater than 34 days (or slightly more than 10 menstrual cycles per year) would be considered to be suffering from 'oligomenorrhea'. However, many studies use a stricter definition of oligomenorrhea of eight or fewer menstrual cycles per year, which translates into menstrual cycles that are at least 45 days in the interval". Several sub-clinical forms of ovulatory dysfunction are not included in this diagnosis which acts as an area of error.

Polycystic Ovaries as A Criteria- "Adams and colleagues defined polycystic ovaries by transabdominal ultrasonography by the presence of at least 10 cystic areas and measuring 2-8 $\mathrm{mm}$ in diameter in one plane of at least one ovary, arranged peripherally around a dense core of ovarian stroma, or scattered throughout an increased amount of stroma. More recently, polycystic ovaries have been defined by the presence of at least 12 such areas measuring $2-9 \mathrm{~mm}$ in diameter". The inconsistencies in the number and size of the cysts offer room for error as seen in the previous observations.

\section{Causative Agents of PCOS [Figure 1]}

Genetic Influence- PCOS exhibits a familial pattern of inheritance. "The genes that have been nominated as candidates tend to fall into four different categories; those related to insulin resistance, those related to androgen biosynthesis and actions, those responsible for inflammatory cytokine responses, and others. Although the genes are related to commonly exhibited biochemical factors, they do not follow the Mendelian pattern of inheritance. According to genome-wide association studies, there are a total of 16 loci associated with PCOS discovered so far. "The genetic loci related to PCOS have links to neuroendocrine, metabolic, and reproductive pathways and links with the genetic associations of menopause, metabolic disorders, depression, and malepattern balding".

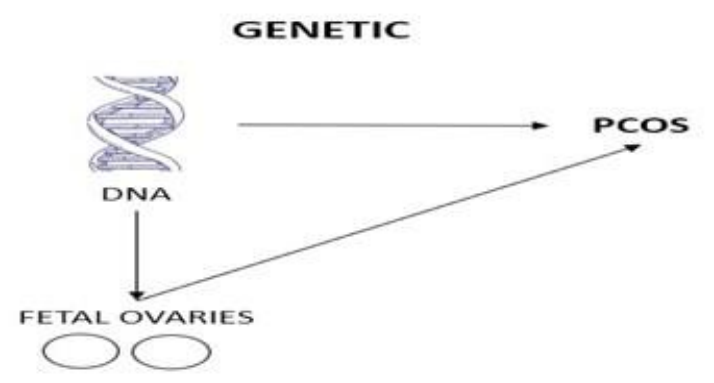

Figure 1: Potential sources of PCOS. AMH indicates anti-Mullerian hormone; PCOS, polycystic ovarian syndrome. 
Epigenetic Influence- So far, we have discussed that external factor can be a causative agent for PCOS. "One plausible theory is that hyper exposure to androgens in utero programs the expression of fetal genes, which then destine the fetus to develop polycystic ovaries, hyperandrogenism, insulin resistance, and hormonal profiles in keeping with the syndrome in adult life". The Barker hypothesis supported this by showcasing a link between birth weight and cardiovascular diseases. The theory of the influence of hyperproduction of androgen in PCOS patients comes mostly from animal trials performed on sheep and monkeys. Sheep were chosen to be excellent subjects for the clinical trials and were administered with doses of androgen. They were found to have lower levels of primordial follicles, higher numbers of primary follicles, higher numbers of antral follicles, the persistence of follicles, and increased follicular recruitment. Insulin resistance was also a major factor monitored in the subjects. Abbott et al experimented-on rhesus monkeys and on injecting pregnant monkeys with testosterone, symptoms similar to PCOS in humans were observed.

\section{Studies Conducted in the Past}

\section{The First Diagnostic Criteria}

Formal diagnostic criteria were proposed and afterward largely utilized for PCOS. It was not until the early 1990s at a National Institute of Health (NIH) sponsored conference on PCOS that formal diagnostic criteria were proposed and afterward largely utilized, named as Rotterdam Criteria. It was established by a group of experts during a conference in Rotterdam held in 2003 as mandatory. The criteria incorporated the size and morphology, as determined by an ultrasound, of the ovary into the diagnostic criteria. It seemed that the ovarian stroma area to total area ratio had been the best condition of a PCOS diagnosis. Almost one-quarter of the population had the appearance of polycystic ovaries when examined ultrasonically, but more than half of these had no clinical signs or symptoms. These women are referred to have polycystic ovaries. Increased $\mathrm{LH}$ and testosterone levels were regarded to be of key importance in diagnosing PCOS. Many years have passed since the first publication concerning PCOS, but the etiology of PCOS is still puzzling. It is now accepted that it is multifactorial and partly genetic; however, several candidate genes have been postulated. In 2006 the Androgen Excess Society (AES) issued a statement criteria attempted to establish hyperandrogenism as a sine qua non-diagnostic condition in combination with other signs of the syndrome. PCOS was described as a distinct masculinization and theca luteinization syndromes, many women met the clinical criteria of PCOS, without confirmation of hormone secretion disorders in laboratory tests. Secretion of hormones released by the pituitary gland and gonads is pulsative, so maximal and minimal concentrations may differ significantly during the day. . Insulin resistance has been noted consistently among many women with PCOS, especially in those with hyperandrogenism, but it is not included in any of the diagnostic criteria. resistance, metformin can be used in the treatment. There have been several recommendations for the use of insulin-sensitizing agents not only to restore ovulation but to facilitate weight loss and counteract androgenic symptoms.

\section{Steroidogenesis and Metformin}

Hyperinsulinemia contributes to the ovarian androgen overproduction and glucose intolerance of polycystic ovary syndrome (PCOS). A study was conducted to determine whether metformin would reduce insulin levels in obese, nondiabetic women with PCOS during a period of weight maintenance and thus attenuate the ovarian steroidogenic response to the $\mathrm{GnRH}$ agonist leuprolide. The subjects had an oral glucose tolerance test, a GnRH agonist (leuprolide) test, a frequently sampled iv glucose tolerance test, graded and oscillatory glucose infusions, and a dual-energy x-ray absorptiometry scan before and after treatment with metformin. Weight maintenance, oral glucose tolerance, insulin sensitivity, and the relationship between $\mathrm{Si}$ and first-phase insulin secretion (AIRg vs. Si) were not improved by metformin. The insulin secretory response to glucose, administered in both graded and oscillatory fashions, was likewise unaltered in response to metformin. Free testosterone levels remained about 2-fold elevated. Both basal and stimulated $\mathrm{LH}$ and FSH levels were unaffected by metformin. The mean responses to leuprolide of 17-hydroxyprogesterone as well as those of the other ovarian secretory products (androstenedione, dehydroepiandrosterone, progesterone, and estradiol) were not attenuated by metformin.

\section{Diet and Physical Activity in PCOS}

Polytomous logistic regression analyses examined the associations between diet and PA with PCOS, HA, and OA status (outcomes), adjusting for age, race, total energy intake, education, and/or body mass index. The threshold for statistical significance was set at $p<0.05$. The mean age of the participants was 25.4 years (SD 3.6) and $46.8 \%$ of participants were Black women. There was little to no association of total energy intake, nutrients, diet quality, and PA with PCOS, HA, or OA status. Energy intake, nutrient composition, diet quality, and PA were not associated with PCOS, supporting recent PCOS guidelines of using national recommendations for the general population to encourage health-promoting behaviors among women with PCOS. Energy intake, nutrient composition, diet quality, and PA were not associated with PCOS, supporting recent PCOS guidelines of using national recommendations for the general population to encourage health-promoting behaviors among women with PCOS. The combined involvement of oligomenorrhea (OA) and hyperandrogenism (HA) is used by the National Institutes of Health $(\mathrm{NIH})$ to describe a relatively homogeneous patient population with the highest risk of reproductive and metabolic dysfunction. The Rotterdam criteria define PCOS by the combined presence of two out of three cardinal features: $\mathrm{OA}, \mathrm{HA}$, and polycystic ovarian morphology; the Rotterdam criteria captures a more 
heterogeneous patient population, including mild PCOS phenotypes (i.e., women with regular menstrual cycles and $\mathrm{HA}$ or women with $\mathrm{OA}$ and normal androgens). Milder phenotypes could indicate different etiologies and lower risks of metabolic and reproductive complications. The existence of isolated $\mathrm{OA}$ versus $\mathrm{HA}$ and/or the additive effects of these two features in combination with adiposity may explain differences in metabolic and reproductive risk across PCOS phenotypes. Extreme PCOS phenotypes have been shown to have more adiposity than moderate PCOS phenotypes. So, it can be said that current dietary and physical activity guidelines for women with PCOS are based on those for the general population or other clinical populations (e.g., diabetes mellitus), and do not take into account the complex biology and/or psychosocial factors associated with PCOS $[6,7]$. Understanding the links between dietary consumption and physical activity levels in people with PCOS is crucial. An understanding of associations between dietary intake and physical activity levels with PCOS is ultimately needed to determine the relevance of tailored therapies for this patient population.

\section{PCOS and Quality of Life}

HRQOL is a self-perceived health status as a consequence of any disease that is measured by a health status questionnaire. ${ }^{29} \mathrm{PCOS}$ questionnaire has reasonable internal reliability, good test-retest reliability, good concurrent and discriminant validity, and a reasonable factor analysis making PCOS questionnaire a useful and promising tool for HRQOL in PCOS cases. This prospective case-control study was conducted among 100 PCOS and 200 healthy control cases attending tertiary care set up by AlIMS, Patna during 2017 and 2018. Prevalidated questionnaires like Short Form Health survey-36 were used for evaluating the impact of PCOS in women. Multivariate analysis was applied for statistical analysis. In PCOS cases, socioeconomic status was comparable in comparison to healthy control. After checking for the normality condition for continuous variables, the appropriate statistical test was applied. Confounders like excessive body weight were taken into consideration. Quantitative data expressed as mean $\pm S D$, minimum and maximum followed normal and skewed distribution respectively. Analysis of the covariance model (ANCOVA) was used to address potential confounders. Pearson Chi-Square test and Fisher exact test were used to check the association between qualitative variables and categorical variables. Logistic regression analysis was used to estimate odds $(95 \% \mathrm{Cl})$ and models were robust for PCOS and other variables. Multivariable linear regression analysis was performed to observe the association between the variables. Independent t-test and One Way ANOVA used to compare normally distributed continuous variables between two and three categories respectively. Rank sum/Kruskal Wallis test used for comparing skewed continuous variables among categories and to look for associations between demographic categories. The women with PCOS and HC were comparable in respect of marital status and family type. Statistically significant differences were observed between $P C O S$ and $H C$ in terms of age $(P<0.020)$, BMI $(P<0.001)$, educational status $(P<0.001)$, marital status $(P>0.05)$ and work category $(P<0.001)$. Total $97 \%$ of $P C O S$ cases were below the age of 30 years in comparison to $78 \%$ of control. Among all PCOS cases, $60 \%$ were students and almost $54 \%$ received higher education. Among the $\mathrm{HC}$ group, $39 \%$ were students and only $15 \%$ received higher education $(\mathrm{P}<0.001)$. A higher percentage of PCOS cases $(16 \%)$ belong to greater BMI (>30) in comparison to $\mathrm{HC}(2 \%)$. Here, PCOS cases showed significantly decreased HRQOL. The higher age of menarche, irregular/delayed menstrual history, absence of a child, were significantly altered in PCOS cases than control. The number of children, frequency of pregnancy, and miscarriage were also observed higher in PCOS cases. Furthermore, in various categories of age, $B M I$, educational status, and marital status, significant differences were observed in the different domains of SF36 between PCOS and healthy control. Altogether, increased BMI, menstrual irregularities, educational status and marital status play a major role in altering $\mathrm{HRQOL}$ in PCOS cases and psychological care must be given during patient care.

\section{Hypertension and PCOS}

Researchers conducted a secondary analysis of longitudinal data from the Australian Longitudinal Study on Women's Health intending to evaluate the incidence of hypertension among women with and without PCOS over 15 years. Findings suggest a higher likelihood to develop hypertension among women with PCOS from early adulthood; this was observed independent of BMI, which is further exacerbated by obesity. They suggest the inclusion of PCOS in hypertension risk stratification assessments as possibly beneficial for early identification of the disorder. The incidence of hypertension was significantly higher among women with PCOS compared to women without. There were significant differences in time to hypertension development between women +/- PCOS. Hypertension was observed among women with PCOS from early adulthood and across BMI categories. The difference in the actual number of incident hypertension cases between women with and without PCOS was fourfold higher among women who were obese at baseline, compared to age-matched lean women. PCOS was independently associated with hypertension with a $36 \%$ greater risk, adjusting for $\mathrm{BMI}$ and other confounders. 


\section{Diagnosis}

Table 2: Differential diagnosis of PCOS

\begin{tabular}{|c|c|c|c|}
\hline Diagnosis & Pathology & Clinical Presentation & Testing \\
\hline $\begin{array}{c}\text { Congenital adrenal } \\
\text { hyperplasia (adult onset) }\end{array}$ & $\begin{array}{c}\text { Heterozygous } 21 \text { hydroxylase } \\
\text { enzyme deficiency in the adrenals }\end{array}$ & Hirsutism and Irregular Periods & 17 hydroxyprogesterone \\
\hline $\begin{array}{c}\text { Prolactinoma } \\
\text { Cushing's syndrome } \\
\text { Cushing's disease }\end{array}$ & Pituitary adenoma & Amenorrhea and Galactorrhoea & Prolactin \\
\hline Idiopathic hirsutism & Adrenal adenoma & Hirsutism and Oligomenorrhea & Cortisol, DHEA-S, ACTH \\
\hline
\end{tabular}

There are certain basic diagnosis methods undertaken to detect PCOS in women [Table 2]:

- Phenotypic Analysis- The presence of unwanted or excess facial or body hair growth.

- Diagnosis of Hyperandrogenism- It has been reported that $78 \%$ of PCOS patients exhibit hyperandrogenism. The clinical method of diagnosing hyperandrogenism is assessing hirsutism, alopecia, and acne. Hirsutism is assessed using the Ferriman Gallwey Score (requirement $>$ / 4 to 6), alopecia is done using the Ludwig Visual Score but there is no specific requirement for acne. In the absence of clear measurements, free testosterone, free androgen index, and bioavailable testosterone levels should be calculated. LC-MS techniques are recommended for precise results. In the absence of accurate testosterone measurements, DHEAS can also be considered.

- Diagnosis of Polycystic Ovaries- Ultrasound is the most precise and detailed means of getting information about the presence of polycystic ovaries in the patient but it is not recommended in patients having a gynecological age of fewer than 8 years post menarche. When using ultrasound to diagnose, the observation should be (1) more than 20 cysts/follicles of size $2-9 \mathrm{~mm}$ (2) ovarian volume $>/ 10 \mathrm{~mL}$. When not using ultrasound, Anti-Mullerian Hormone is taken as a suitable biomarker for diagnosis.

- Diagnosis of Irregular Menstruation- On average, 85$90 \%$ of women with oligomenorrhea and $30-40 \%$ of women with amenorrhea have PCOS. "Ovulatory dysfunction is clinically reflected by irregular menstrual cycles, which are defined as shorter than 21 days or longer than 45 days in women between 1and 3-years post-menarche and less than 21 or more than 35 days in women over 3 years post menarche to perimenopause". ${ }^{22}$

Exclusion of-

1. steroid 21-hydroxylase (CYP21A2) deficient nonclassic adrenal hyperplasia (NCAH) by calculating follicular phase serum 17-hydroxyprogesterone (17OHP) level,
2. hyperprolactinemia and thyroid dysfunction by serum prolactin and TSH measurements,

3. androgenic drugs by clinical evaluation ${ }^{10}$

Controversies Surrounding the Existing Diagnosis:

Irregularities In Cycles and Ovarian Dysfunction-

- It is normal for people under stress and those who indulge in strenuous activities to have irregular menstrual cycles.

- Several people take contraceptive measures which hinder the normal cycles.

- Different people have cycles of different lengths due to which the time appropriate for testing is delicate.

- $\quad$ "If the menstrual cycles are very irregular, measuring serum progesterone is futile as the diagnosis is based on clinical features and ovulation induction therapy is indicated".

- The term "irregular cycles" is difficult to define in patients undergoing puberty.

- In adolescents, setting a clear-cut boundary between PCOS and normal physiological immaturity of the hypothalamic-pituitary axis is controversial.

- "The European Society of Human Reproduction and Embryology (ESHRE) international evidence-based guidelines recommendations around irregular cycles in adolescents are based on pediatric consensus opinion. This consensus recommends that if an adolescent girl has irregular periods (<21daysor $>35$ days) even after 3 years of menarche, she should be assessed for PCOS".

- Problems With Biochemical Diagnosis of Hyperandrogenism-

- The androgens usually measured are free testosterone, total testosterone, and bioavailable testosterone but only $1-3 \%$ of testosterone is unbound to plasma proteins.

- An alternative method of measurement is the use of FAI but there are inaccuracies since it also involves the measurement of testosterone. 
- Total testosterone values are inconsistent since they change with the time of the day, age of the patient, and also because of the medication the patient is on.

- Furthermore, there is no universally accepted testosterone calibrating standard.

- Women are usually under contraceptives which need to be discontinued for at least 3 months before testing.

- LC-MS assays used for the measurement during diagnosis are also known to be unreliable.

- Hirsutism is a great indicator of hyperandrogenism in women suffering from PCOS but there are inconsistencies in its diagnosis as well since (1) An overestimation of hirsutism can easily be made if body and facial vellus hair is wrongly perceived as terminal hair (2) Patients tend to treat excess hair growth before coming in for testing (3) the cut-off value for diagnosis differs with ethnicity.

- Inconsistency In Diagnosis of Polycystic Ovaries-

- The polycystic ovaries in PCOS can be confused with other causes of multi follicular ovaries which could be both physiological (puberty) and pathological.

- PCOS cannot be diagnosed using ultrasound in adolescents.

- Setting a number and size threshold for antral follicles is controversial since they vary with age and ethnicity.

- Inconsistencies in the method of counting due to observer variability.

- Different ultrasound machines can provide different images which brings forth the question of the reliability of 3D ultrasound.

- Other biomarkers such as ovarian stroma and ovarian blood flow have been rejected as diagnostic tools.

- Although several tests and studies have been done to use serum Anti Mullerian Hormone as a non-invasive biomarker, it is yet to be accepted. ${ }^{26}$

- Emerging Biomarkers:

- Insulin as a Biomarker-Insulin resistance is one of the most prevalent features of PCOS patients and it is characterized by the need of the body for an increased amount of insulin to perform the basic tasks. Inflammation is a common symptom of PCOS patients and it is proposed that inflammation, along with obesity is one of the main causative agents of insulin resistance in PCOS patients. Insulin resistance is closely related to blood glucose levels due to which parameters such as fasting-glucose-to-insulin ratio, homeostatic model assessment, and quantitative insulin sensitivity check index can be used as diagnostic tools for PCOS. The reason behind the close relationship between insulin and glucose is that insulin stimulates glucose uptake in the body by increasing the translocation of GLUT4, which is a crucial transmembrane glucose transporter. Several in-vitro studies have been done on adipocytes and skeletal muscles to research the mechanism of insulin and its involvement in human biochemistry. ${ }^{19}$

- Amh as a Biomarker- "In healthy women, serum AMH levels are slightly increased during childhood, reach a maximum and remain stable at puberty, then decrease and become undetectable in the serum of menopausal women. In PCOS women, serum AMH is increased with antral follicle count (AFC), LH, testosterone (T), and estradiol while it decreases with $\mathrm{BMI}$, age, and $\mathrm{FSH}$ in infertile control women". Kedem et al. suggested in PCOS patients, AMH downregulation in late antral stages is impaired and high levels of serum AMH may originate from the higher number of small antral follicles. Studies have found that AMH levels are related to both anovulation and hyperandrogenism. Patients suffering from PCOS experience an increase in the frequency of the $\mathrm{GnRH}$ hypothalamic pulses due to which the baseline and stimulated concentrations of $\mathrm{LH}$ are higher thus subsequently increasing $\mathrm{AMH}$. It is postulated that PCOS patients with high $A M H$ inhibited the secretion of progesterone and the low level of progesterone promoting GCs apoptosis; these effects might be assumed to inhibit folliculogenesis and may have an influence on the pregnancy outcomes of PCOS patients. ${ }^{17}$

- Calprotectin as a Biomarker- A clinical trial was conducted to research the same and the details have been discussed below.

Experiment

\section{Subjects Used [Table 3]-}

A total of 95 women were enrolled from the Obstetrics and Gynecology Hospital of Fudan University, China, between April 2013 and December 2014. Out of these, 41 were newly diagnosed PCOS patients and 54 were healthy individuals having no history of diabetes, hypertension, hyperprolactinemia, thyroid disease, Cushing's syndrome, or congenital adrenal hyperplasia. "Subjects taking drugs such as insulin-sensitizing drugs, oral contraceptives, corticosteroids, anti-androgens, and gonadotropinreleasing hormone agonists or antagonists were excluded from the study." The polycystic ovarian syndrome was diagnosed according to the criteria developed by the PCOS Consensus Workshop Group in 2003 as follows:

1. Polycystic ovaries, identified by ultrasound ( 512 small follicles with the diameter of 2-9 $\mathrm{mm}$ in at least one ovary, and/or ovary volume $>10 \mathrm{~mL}$ )

2. Clinical or biochemical evidence of excessive testosterone (testosterone $52.55 \mathrm{mmol} / \mathrm{L}$ ).

3. Anovulatory menstrual disorder.

Two of the above criteria with the exclusion of other causes were sufficient to diagnose PCOS". 
Table 3: Main clinical and hormonal features in females with PCOS and females without PCOS

\begin{tabular}{|c|c|c|c|}
\hline & $P \operatorname{COS}(n=41)$ & Non-PCOS $(n=54)$ & $\mathbf{P}$ \\
\hline Age (years) & $26.67 \pm 4.51$ & $27.33 \pm 4.78$ & 0.88 \\
\hline BMI (kg/m?) & $23.67 \pm 3.06$ & $22.83 \pm 3.47$ & 0.79 \\
\hline Blood pressure systolic $\mathrm{mmHg}$ & $121.73 \pm 12.87$ & $119.33 \pm 16.03$ & 0.87 \\
\hline Blood pressure diastolic $\mathrm{mmHg}$ & $82.2 \pm 10.57$ & $81.03 \pm 13.94$ & 0.93 \\
\hline $\mathrm{LH}(\mathrm{mlU} / \mathrm{mL})$ & $8.7 \pm 1.71$ & $4.3 \pm 0.57$ & 0.03 \\
\hline $\mathrm{FSH}(\mathrm{mlU} / \mathrm{mL})$ & $5.03 \pm 1.36$ & $9.97 \pm 1.68$ & 0.03 \\
\hline LH/FSH ratio & $1.73 \pm 0.42$ & $0.42 \pm 0.15$ & 0.02 \\
\hline SHBG (nmol/L) & $33.67 \pm 7.51$ & $61.34 \pm 10.33$ & 0.04 \\
\hline DHEAS (mg/L) & $2.4 \pm 0.36$ & $1.27 \pm 0.25$ & 0.02 \\
\hline Testosterone (wg/L) & $0.62 \pm 0.23$ & $0.23 \pm 0.06$ & 0.09 \\
\hline Fasting glucose (mg/dL) & $88.83 \pm 10.52$ & $89.6 \pm 9.74$ & 0.94 \\
\hline Fasting insulin (U/L) & $11.1 \pm 1.73$ & $6.7 \pm 1.39$ & 0.04 \\
\hline HOMA index & $2.42 \pm 0.33$ & $1.46 \pm 0.17$ & 0.02 \\
\hline \multicolumn{4}{|l|}{ Results are average \pm SD } \\
\hline
\end{tabular}

- Tests Conducted- Periodic biochemical and physical examinations were conducted on the subjects and included the measurements of BMI, fasting serum glucose and insulin, luteinizing hormone (LH), folliclestimulating hormone (FSH), dehydroepiandrosterone sulfate (DHEAS), and Sex Hormone Binding Globulin (SHBG) concentrations, etc. The calprotectin concentrations were analyzed using ELISA.

- Observations- "The females' ages, BMI values, blood pressure, and fasting glucose levels were similar in PCOS and control groups. Fasting insulin, HOMA-IR, DHEAS, LH concentrations, and LH/ FSH ratio were significantly higher, whereas FSH and SHBG concentrations were lower in the PCOS group compared with controls". As expected, the calprotectin levels were much higher in PCOS patients, about $3.5+/-1.5 \mathrm{~g} / \mathrm{mL}$ as compared to 1.4 +/_ $0.8 \mathrm{~g} / \mathrm{mL}, \mathrm{P}<0.01$ in normal candidates [Figure 2]. ${ }^{20}$

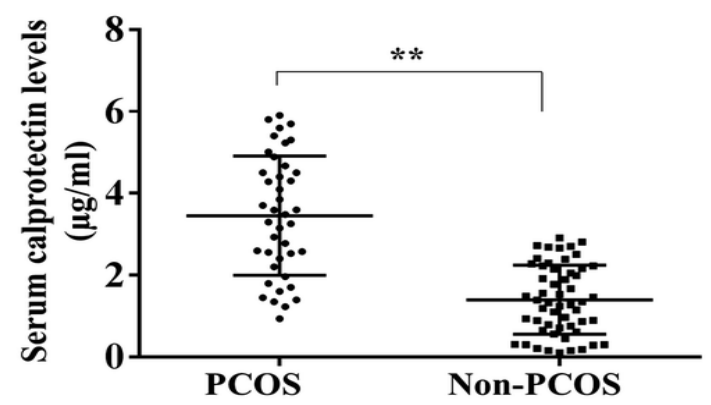

Figure 2: Serum calprotectin concentration in PCOS and non-PCOS groups. Calprotectin concentrations were measured in serum from patients with PCOS $(n=41)$ and without PCOS $(n=54)$. Data are presented as mean \pm SD. $* *, P<0.01$.
- $\quad$ mRNA as a Biomarker- A study on mi-RNA 93 and 223 was approved by The Newcastle and North Tyneside Ethics Committee, the details of which have been mentioned below.

- Participants -

- 25 non-medicated women between the age of 18-45 suffering from PCOS recruited from local PCOS biobank

- 25 normal women between the age of 20-44 with matching age and BMI as the patients from the same biobank

- All the participants were Caucasian, non-smokers, and not on any contraceptive methods.

- The healthy patients had regular periods, no significant medical history, did not have non-alcoholic fatty liver disease, and had no signs of hyperandrogenemia.

- $\quad$ All the participants were tested for non-classical 21hydroxylase deficiency, hyperprolactinemia, Cushing's disease, and androgen-secreting tumors. Diabetes was also excluded by a $75 \mathrm{~g}$ oral glucose tolerance test.

- None of the patients had successful pregnancies or miscarriages at least five years before the study entry.

- Tests Conducted-

Blood samples after overnight fasting

- Serum testosterone was measured using isotope dilution Liquid Chromatography with tandem mass spectrometry (LC-MS) 
- $\quad$ SHBG was calculated using an immunometric assay with fluorescence detection on the DPC Immulite 2000 analyzer. The free androgen index was quantified by multiplying total testosterone with 100 and dividing by SHBG levels.

- Serum insulin was assayed using a competitive chemiluminescent immunoassay performed on the manufacturer's DPC Immulite 2000 analyzer with analytical sensitivity $=2 \mu \mathrm{U} / \mathrm{mL}$ and coefficient of variation $=6 \%$

- The plasma glucose was measured using a Synchron LX 20 analyzer with the coefficient of variation $=1.2 \%$ and mean glucose value $=5.3 \mathrm{mmol} / \mathrm{L}$

- Insulin resistance was calculated using the Homeostatic model assessment (HOMA) method [HOMA-IR $=($ insulin $\times$ glucose $) / 22.5$ ], and pancreatic $\beta$ cell sensitivity measured by HOMA- $\beta$ [HOMA- $\beta=(20 \times$ insulin)/glucose -3.5].

- $\quad$ Serum CRP (c-reactive protein) was calculated using Beckman DXC Analyzer.

- Total RNA was extracted from the plasma using the MiRVana PARIS kit. The RNA was then stored at $-80^{\circ} \mathrm{C}$.

- Total RNA was reverse transcribed using a Reverse Transcription Kit and then amplified in a 96 well StepOnePlus Polymerase Chain Reaction (PCR) System using a mature mi-RNA TaqMan assay using the Taqman 2x Universal PCR master mix, No AmpErase UNG (Life Technologies), with each reaction performed in triplicate.

- $\quad$ Analysis Results [Table 4]-

- $\quad 8 / 25$ patients suffering from PCOS had a BMI of equal to or less than $25 \mathrm{~kg} / \mathrm{m} 2$ and only 1 of them had elevated Free Androgen Index (FAI).

- In the PCOS group insulin was increased $(p=0.03)$, HOMA-IR did not differ $(p=0.07)$, there was a

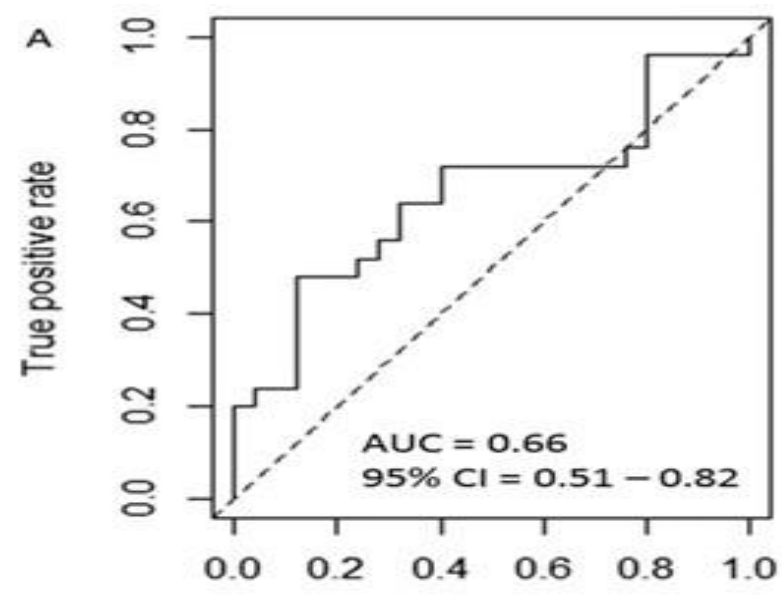

False positive rate significant increase in HOMA- $\beta(p=0.001)$ and testosterone was elevated $(p=0.03)$.

- The expression of both miRNA-93 and miRNA-223 was much higher in PCOS $p(p=0.009$ and $p=0.029$, respectively)

- No correlations were found between the two mi-RNA and they were also found independent of any metabolic and hormonal parameters.

- The diagnostic values were calculated using the Reactive Operative (RoC) curve and calculation of the area under the curve [Figure 3].

- It was seen that miR-93 is a more efficient biomarker for the diagnosis of PCOS. Multiple logistic regression and ROC curve analysis were used to determine whether the combination of mi-RNA 93 with mi-RNA 223 had improved diagnostic potential over mi-RNA 93 alone but no improvements were recorded.

- Target gene prediction was done using miRWalk, miRanda, TargetScan, and DIANA, and a list of possible target genes were identified.

\section{Analysis Discussion}

From the above findings we can conclude that although there is no definitive test for the diagnosis of PCOS, we can still set certain parameters and their detailed requirements to help with the diagnosis. Furthermore, the inconsistency in the characteristics of the patients from different regions can be written down as the major reason for the error in diagnosis. This can be solved by setting different "ranges" and standards for PCOS diagnosis in different areas of the world. Although unusual, instead of having universal criteria, this method can be utilized for precise diagnosis. So far, even though the diagnostic tools and tests are insufficient, alternative biomarkers are yet to be approved globally for a better diagnosis but AHM seems to be the most promising. ${ }^{14}$

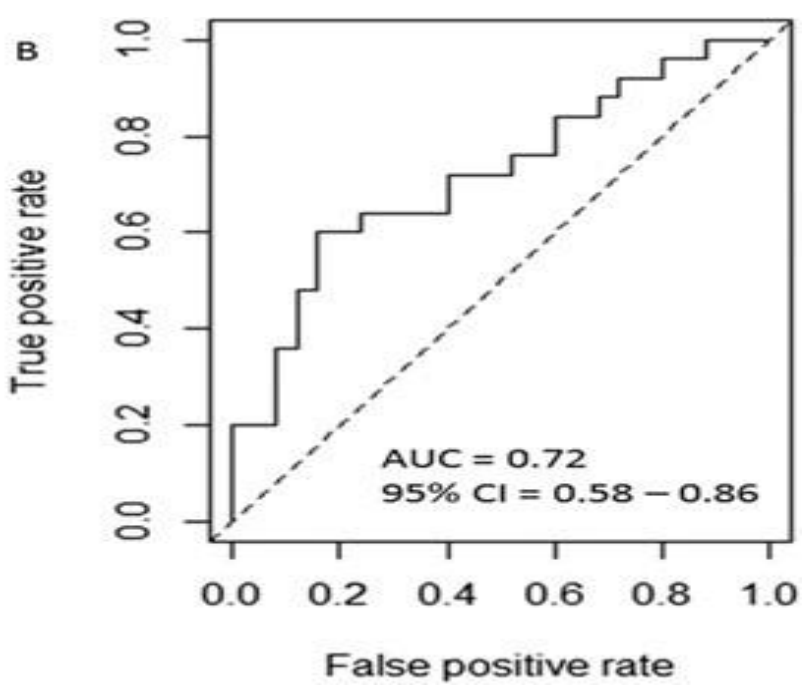

Figure 3: ROC curve analysis of (A) miR-223 and (B) miR-93 to discriminate women with PCOS from healthy controls. 
Table 4: Demographics, biochemical and clinical markers for the PCOS and control group (unpaired t test) (BMI, body mass index; FAI, free androgen index; SHBG, sex hormone binding globulin; HOMA-IR, homeostatic model assessment-insulin resistance; HOMA- $\beta$, homeostatic model assessment-insulin beta cell sensitivity; hsCRP, high sensitivity C-reactive protein.)

\begin{tabular}{|c|c|c|c|}
\hline & Normal $\mathbf{n = 2 5}$ & PCOS $\mathbf{n = 2 5}$ & P value \\
\hline Age (years) & Mean (SD) & Mean (SD) & 0.97 \\
\hline Weight (kg) & $32.2(7.7)$ & $32.1(9.0)$ & 0.79 \\
\hline BMI (kg/m2) & $76.0(18.8)$ & $77.4(16.3)$ & 0.31 \\
\hline Fasting glucose (mmol/l) & $27.1(5.8)$ & $28.8(5.4)$ & 0.56 \\
\hline 2 Hour glucose (mmol/l) & $4.7(0.4)$ & $4.8(0.6)$ & 0.06 \\
\hline Androstenedione (nmol/I) & $4.9(1.2)$ & $5.7(1.3)$ & 0.25 \\
\hline ALT (IU/L) & $8.4(5.1)$ & $10.7(6.7)$ & 0.79 \\
\hline Insulin (U/ml) & $24.3(14.4)$ & $23.3(12.7)$ & 0.03 \\
\hline HOMA-IR & $6.8(3.7)$ & $10.2(6.4)$ & 0.07 \\
\hline HOMA-B & $1.5(0.9)$ & $2.3(1.7)$ & 0.001 \\
\hline Testosterone (nmol/L) & $38.4(66.1)$ & $158.8(89.7)$ & 0.03 \\
\hline SHBG (mmol/L) & $1.2(0.7)$ & $2.3(1.6)$ & 0.29 \\
\hline FAl & $81.8(105.8)$ & $52.6(53.1)$ & 0.13 \\
\hline hsCRP (mg/1) & $2.6(1.7)$ & $11.1(18.9$ & 0.65 \\
\hline miR-93 expression (relative to control group) & $2.3(3.8)$ & $2.8(3.9)$ & 0.009 \\
\hline miR-223 expression (relative to control group) & $1.0(0.6)$ & $2.0(1.6)$ & 0.029 \\
\hline
\end{tabular}

\section{Treatment}

Women with PCOS show various kinds of clinical features including hyperandrogenism with or without skin manifestations, irregular menses (anovulation or oligoovulation), absence of other androgen disorders (adrenal hyperplasia or tumor), and polycystic ovaries on ultrasonography. However, hyperandrogenism remains a telltale feature of PCOS. The treatment strategies of PCOS are directed towards the suppression of excessive androgen levels in the body with the improvement of hirsutism and acne, restoration of anovulation, and diminishing the risk of metabolic disorders like Type 2 Diabetes Mellitus and other cardiovascular diseases. Insulin resistance is the prominent feature of PCOS, which can be a result of the expression of thrifty genes like Xbal poly- morphism of the glycogen synthase gene, the T228A polymorphism of the SaRBS I gene, and the D85 and Y85 variants of the UGT2B 15 gene in a not so healthy environment of plentiful food and no exercise. ${ }^{4}$ The treatment for PCOS largely depends on the severity of an individual's symptoms. Surgical procedures, pharmacological medications, lifestyle changes, etc can improve the symptoms and delay the complications associated with PCOS. Treatment for PCOS generally follows a multidisciplinary, biopsychosocial approach decided by the medical needs and the desires of the patient. ${ }^{11} \mathrm{~A}$ single treatment tone treats only a particular abnormality of PCOS. This review summarizes the current treatment strategies for PCOS and some other promising effective treatment modes in the future.

\section{Lifestyle change}

PCOS and its clinical features are seen more regularly in women who are overweight and obese as compared to women of normal weight. It is more common in women with PCOS. Modest weight loss has proven to improve almost all the anomalies associated with PCOS like reproductive, metabolic, hormonal, lipid profile, as well as psychological parameters. ${ }^{11}$ In obese adult women, a 5- 10 $\%$ reduction in weight reported lower hyperandrogenism as well as lower fasting insulinemia and improved ovulation. Weight loss improves every parameter of PCOS, restoring ovulation, decreasing testosterone levels while raising Sex Hormone Binding Globulin (SHBG) levels. Hence the most preferred and most effective method of treatment for PCOS is lifestyle interventions. Effective exercise and nutritional counseling are important for adolescents to overcome the morbidities of PCOS and to have a good quality of life ahead. Certain cardio exercises and brisk walking have proven to reduce waist to hip ratio and homocysteine levels- an indicator of cardiovascular risks. ${ }^{4}$ Regular exercising also improves insulin resistance and regularises the menstrual cycles. ${ }^{4,11}$ Weight loss restores ovulation, decreases insulin levels, diminishes acanthosis nigricans, lowers testosterone levels while raising Sex Hormone Binding Globulin levels. ${ }^{4}$ Obesity affects the birth rate and the body's total response to pharmaceutical treatments for infertility. Diet with a low glycemic index and modifying the intake by increasing the protein to carbohydrates ratio is proven to be beneficial for obese women. ${ }^{4}$ A study conducted by Panidis et al recommended that patients who are overweight and 
diagnosed with PCOS should have control over their diets by consuming approximately 1200 - 1500 calories per day and by spending some time in moderate exercises at least 30 minutes per day. ${ }^{23}$ By doing so, alongside the drug treatments helped do good to insulin levels, testosterone levels and reducing menstrual disorders. Another study conducted by Nybacka et.al also proved similar results. ${ }^{23}$ By restricting the total calorie intake and taking part in moderate exercises, reducing BMI to normal range, and giving up smoking and drinking can improve insulin resistance and free testosterone levels, reduce menstrual disorders, hirsutism, and other symptoms. ${ }^{23}$ The clinical experiences have shown that lifestyle intervention produces positive results only in the highly motivated adolescent with PCOS who has the support and commitment of the family. However, this mode of therapy alone produces limited results. Overall successful lifestyle change and weight loss can prove beneficial in combination with pharmacological treatments. ${ }^{4}$ Despite the well-known benefits of weight loss, the attrition rate in lifestyle modification is quite high. This can be associated with psychological problems such as depression and behavioral changes.

\section{Oral Contraceptive Pills (OCPS)}

Oral contraceptive pills are used as first-line treatment for long-term management of PCOS. OCPs with nonandrogenic progestin quell ovarian functions and restores menstrual cycles. These pills are proven useful to attenuate hirsutism and acne; when prescribed along with metformin increases the bone mineral density, reduces the risk of ovarian and endometrial cancers, and fends off anemia. Anti-androgens like cyproterone acetate can also be used. ${ }^{2}$ Greek investigators have reported that $150 \mathrm{mg}$ of desogestrel when combined with 30ug Ethinyl Estradiol lowers the androgen levels, regulates menses, and decreases hirsutism in adolescents. ${ }^{5}$ Although OCPs are known to raise triglyceride levels and total cholesterol levels, it was well tolerated as the LDL/HDL ratio remained constant and there was no increase in body weight nor body fat in women. The Oral Contraceptive Pills (OCPs) which are mainly made up of estrogens and progesterone increases the concentrations of Sex Hormone Binding Globulin, thereby reducing the free testosterone levels, restoring menstrual cycles, reducing hyperandrogenism, and protecting endometrium help dissipate the risk of cancer. Long-term use of OCPs can increase the risk of venous thrombosis. ${ }^{23}$ Some studies have found that Ethinyl estradiol has stronger effects on liver metabolism than the natural estradiol produced in the body. Patients with thrombophlebitis, migraine, and diabetes mellitus are not advisable to go with this mode of treatment or should be only at lower doses after careful considerations. ${ }^{5}$

\section{Insulin Sensitizing Drugs}

\section{Metformin.}

Insulin resistance is the foremost feature of polycystic ovarian syndrome which is also associated with increased cardiovascular risks. Metformin, an insulin-sensitizing drug has transpired as an important integrant of PCOS treatment. It is known to improve insulin sensitivity, glucose metabolism and reduces hyperandrogenism, menstrual irregularities, total cholesterol, LDL cholesterol, and triglycerides while increasing the HDL cholesterol and serum SHBG on the other hand. ${ }^{4}$ Metformin is an effective option to treat both adolescent and adult women with PCOS, although higher success rates at normalizing menses and reducing hirsutism are seen in the younger group of patients. It came out that lean PCOS women did not benefit from metformin in terms of metabolic risk factors. In a 6-monthly study, it was seen that metformin helped improve menstrual irregularities. It was also reported that this modality helped overweight and obese women with PCOS improve fasting glucose, insulin and testosterone levels, BMI, and the frequency of ovulation. ${ }^{7}$ it is postulated that metformin administration, by promoting body-weight loss, can decrease fasting and stimulate plasma insulin concentrations, making this agent helpful in treating insulin resistance. As a result, there is a possibility of a reduced risk of type 2 diabetes and cardiovascular disease in these women. ${ }^{2}$ After 8 weeks of treatment, metformin significantly reduced plasma concentrations of $\mathrm{LH}$, total and unbound testosterone, DHEAS, and increased concentrations of FSH and SHBG. ${ }^{2}$ The mechanism of action involves the reduction in the production of glycogen which improves the sugar metabolism, therefore restoring ovulation $30-50 \%$ of women and mitigating the symptoms of high testosterone to a certain extent. It can be used as a viable option to restore ovulation as no known teratogenic effects were observed. Another study revealed that metformin is also effective to take the edge off the risk of endometrial cancer. ${ }^{11}$ Since metformin regulates ovulation and menstrual cycles as a result it reduces the endometrium exposure to unopposed estrogen thereby dwindling the risk of endometrial cancer. It has to be noted that the use of metformin is lifelong as all the positive results were lost after 3 months of cessation of metformin treatment. ${ }^{4}$ It should be discontinued during pregnancy as it is known to cross the placenta. The review also revealed that a few side effects like vomiting and diarrhea were seen in a few women, but most of them showed better tolerance of the drug without any adverse effects. ${ }^{2}$ Overlooking the duration of treatment, metformin appears to be the main pharmacotherapeutic alternative for PCOS. Much better and cleaner results can be seen if the treatment is given alongside lifestyle modification by changing dietary intake, engaging in weight loss activities and exercises. In conclusion, it is seen that metformin treatment helped improve the metabolic profile of women with PCOS.

\section{Thiazolidinedione (TZD)}

Glitazone treatments show reproductive hormonal effects either by directly affecting the ovary or by reducing the circulating insulin levels. For women who had difficulty responding to metformin, the addition of pioglitazone to their treatment regimen improved glucose/insulin parameters, lowered DHEAS levels, raised SHBG and HDL 
levels, and enhanced cycle regularity. ${ }^{4}$ Certain glitazones like Rosiglitazone and Pioglitazone have exhibited an improvement in insulin sensitivity and decrease in androgen levels. ${ }^{8}$ The TZDs stimulate the peroxisome Proliferator-Activated $\gamma$ receptors and activate the transcription of genes that affect glucose and lipid metabolism. ${ }^{8}$ Glitazone treatments are less relevant in patients with PCOS with a primary complaint of hirsutism. In a contrasting study between Metformin and Glitazone, the BMI of patients treated with pioglitazone remained the same while those treated with metformin showed a decrease in the BMI. ${ }^{5,8}$ Also, the glitazone treatments did not help in the metabolic disorders as compared to its counterpart. This mode of treatment also showed an increase in SHBG levels. Due to the improvement in ovulation, the rates of pregnancy increased, and hence the use of glitazones is recommended only with a combination of OCPs, as studies have revealed fetal loss and fetal growth retardation. ${ }^{8}$ Glitazone also adversely affected the bone mineral density, hence its rationale is still limited for daily praxis.

\section{$\underline{\text { Inositol }}$}

It was later discovered that a defect in the inositol phosphoglycan (IPG) could be a cause of hyperinsulinemia, which formed the basis for new treatment strategies for hyperinsulinemic PCOS patients. ${ }^{16}$ Inositol has been characterized as hexahydroxy cyclohexane which is known to possess 9 stereoisomers. The idea to use inositol as a form of treatment lies in the fact that insulin metabolic signals do not work accurately. ${ }^{16}$

Ovaries of women with PCOS show an increased activity, which leads to conversion of $\mathrm{MI}$ to $\mathrm{DCl}$ and reducing the $\mathrm{MI}$ in the follicles. ${ }^{16}$ Patients treated with Inositol showed improvement in insulin sensitivity and free testosterone concentrations. Additionally, ovulation was also restored in a higher percentage that is $86 \%$ of the PCOS patients. ${ }^{16}$ The use of Myo-Inositol (MYO) in PCOS patients showed improvement in hormonal parameters like Luteinizing hormone, LH: FSH ratio, testosterone, and androgen concentration, and hyperinsulinemia. BMI and menstrual functions improved along with fertility. ${ }^{16}$ Inositols are naturally occurring compounds hence can be considered as an easy and effective treatment.

\section{Surgery}

Surgery proves to be yet another treatment for women with PCOS. Bilateral Ovarian Laparoscopy or Laparoscopic Ovarian Diathermy (LOD) is a kind of surgery available for the treatment of PCOS, which is performed by electrocoagulation or laser perforation, improving the ovarian function by destroying the ovarian stroma, reducing the size of the ovarian matrix, and lowering the production of androgens. ${ }^{4}$ Surgery is not indicated in teenagers, as it does little to improve PCOS symptoms beyond infertility, and pregnancy induction is not desirable in this group. ${ }^{4}$
Apart from LOD, other surgeries performed for the same include Gastric Bypass (GBP), Sleeve Gastrectomy (SG), and Adjustable Gastric Banding ( $A G B$ ). Though Ovarian drilling by diathermy or laser is the most preferred surgical treatment. ${ }^{4}$

\section{Anti- Androgens}

Anti-Androgens have been proven successful in treating hirsutism. Some non -steroidal anti-androgens like Flutamide and Cyproterone acetate (CPA) are widely used for the treatment of hirsutism and acne. ${ }^{5}$ Although no evidence is available regarding the improvement in menstrual regulation, $\beta$ cell functions, and insulin resistance. Another anti- androgen Spironolactone when compared to metformin, showed better performance with regards to hirsutism, acne, menses, and hormonal regulation but did not appreciably improve metabolic idiosyncrasy. Their use should be limited due to proven hepatic toxicity. Moreover, Anti-androgens should not be prescribed during pregnancy due to the possibility of feminizing a male fetus. ${ }^{5}$

\section{Metabolism Related theories}

\section{Vitamin $D$ deficiency theory}

A study conducted by Skowronska et.al. found out that Vitamin D levels in the serum are associated with Sex Hormone Binding Globulin (SHBG). For patients with a deficiency of Vitamin $D$, prescribing the supplements increases insulin sensitivity and lowers total androgen and testosterone levels. ${ }^{4}$

\section{Aromatase Inhibitors}

Clomiphene Citrate (CC) and Letrozole are the current firstline infertility treatment options for patients with PCOS. The study for the use of Letrozole (LE) versus Clomiphene citrate (CC) was conducted with 750 infertile women subjects. ${ }^{12}$ Those women were further subjected to progestin administration to induce menses and were randomly assigned either CC or LE. Live births were higher in patients treated with Letrozole, hence more effective than CC. LE treatment increased the probabilities of ovulation, conception, pregnancy, and live birth. ${ }^{12}$ The authors also concluded that improvement in hyperandrogenism is not obligatory to increase ovulation and birth rate. ${ }^{12}$ Both CC and Letrozole showed side effects like hot flashes and fatigue and dizziness respectively. ${ }^{12}$ These Aromatase inhibitors may be an alternative option for induction of ovulation and pregnancy amongst women with PCOS, but more trials are needed to establish its efficacy.

\section{Herbal Medicines}

The use of Complementary medicine, by women, has been elevated during the past few years and herbal medicine has been the so-called "favorite" treatment option available. Herbal medicines are complex intermediations, known for synergistic and antagonistic interactions. The studies conducted showed that herbal medicines helped 
reduce luteinizing hormone (LH), prolactin, fasting insulin, and testosterone. ${ }^{13}$ There was evidence for the regulation of ovulation, improved metabolic hormone profile, and improved fertility outcomes in patients with PCOS. Tribulus Terrestris, Glycyrrhiza spp., (alone and in combination with Paeonia lactiflora), Paeonia lactiflora (in combination with Cinnamomum cassia) and Cinnamomum cassia demonstrated morphological changes in polycystic ovaries and steroidogenesis, including reduced ovarian volume and cysts, lowered androgens, improved insulin sensitivity and increased oestradiol. ${ }^{13}$ Clinical investigations did not show any adverse effects of the herbal options. Vitex agnus-castus and Cimicifuga racemosa have shown maximum evidence in the management of oligo/amenorrhea and infertility associated with PCOS; and Cinnamomum cassia for improving metabolic hormones in patients with PCOS. ${ }^{13}$ Clinical investigations did not report any adverse effects of these herbal medicines. Hence Herbal medicines can be used as an effective treatment option for women with oligo/amenorrhea and PCOS. The use of herbal medicines of PCOS will require a combination prescription of Glycyrrhiza Spp and Paeonia lactiflora. ${ }^{13}$

In conclusion, even though several treatment options are available, none of them seems to be a magic bullet to treat PCOS as a whole disorder. Several options including metformin, a healthy diet, and exercise are most beneficial. No single drug modality is effective and hence investigators are looking at the effects of combination therapy. In certain cases when the treatment is discontinued, the disease appears to worsen, demanding severe treatment. Thereby careful long-term observation of these treatment options is needed to evaluate therapeutic outcomes.

\section{CONCLUSION}

This research review's purpose is to help the reader understand different aspects posed by the research on Polycystic Ovary Syndrome. This is significant because it gives insights into its prevalence in history, its diagnostic procedures, and the research is done on its treatments. There has been much research and discussion conducted such as surveys, diagnostic procedures, and several types of treatments. Most of the research found was on the lifestyle changes to be made for the cure of this syndrome. More research and testing are required to gain a better understanding of the combination of treatments to be given to properly cure the syndrome for long-term effects.

Acknowledgement: We would like to thank our supervisor/guide Bharat Kwatra, from Invenzion Labs Inc., and our sub-mentor Harsimran Kaur, whose expertise was invaluable in formulating the research questions, methodology, and drawing conclusions. His insightful feedback and guidance pushed us to sharpen our thinking and brought our work to a higher level.

\section{REFERENCES}

1. Duleba AJ, Pawelczyk LA, Yuen BH, Moon YS. Endocrinology: Insulin actions on ovarian steroidogenesis are not modulated by metformin. Human Reproduction. 1993 Aug 1; 8(8): 1194-8.

2. Koch YD, Ernst ME. Use of metformin in polycystic ovary syndrome. Annals of Pharmacotherapy. 2001 Dec; 35(12): 1644-7.

2. Orvieto R, Ben-Rafael Z. Polycystic ovary syndrome: a simplified approach based on the evolving set of symptoms. Human Reproduction. 2003 Apr 1; 18(4): 896-7.

3. Salmi DJ, Zisser HC, Jovanovic L. Screening for and treatment of polycystic ovary syndrome in teenagers. Experimental Biology and Medicine. 2004 May; 229(5): 369-77.

4. Warren-Ulanch J, Arslanian S. Treatment of PCOS in adolescence. Best practice \& research Clinical endocrinology \& metabolism. 2006 Jun 1; 20(2): 311-30.

5. Azziz R. Diagnosing the diagnosis: why we must standardize the defining features of polycystic ovary syndrome:3-5.

6. Cheang KI, Huszar JM, Best AM, Sharma S, Essah PA, Nestler JE. Long-term effect of metformin on metabolic parameters in the polycystic ovary syndrome. Diabetes and Vascular Disease Research. 2009 Apr; 6(2): 110-9.

7. Glintborg D, Andersen M. Thiazolinedione treatment in PCOS-an update. Gynecological Endocrinology. 2010 Nov 1; 26(11): 791-803.

8. Azziz R, Dumesic DA, Goodarzi MO. Polycystic ovary syndrome: an ancient disorder? Fertility and sterility. 2011 Apr 1; 95(5): 1544-8.

9. Goodarzi MO, Dumesic DA, Chazenbalk G, Azziz R. Polycystic ovary syndrome: etiology, pathogenesis and diagnosis. Nature reviews endocrinology. 2011 Apr; 7(4): 219-31.

10. Farrell-Turner KA. polycystic Ovary syndrome: Update on Treatment Options and Treatment considerations for the Future. Clinical Medicine Insights: Women's Health. 2011 Jan; 4: CMWH-S6715.

11. Legro RS, Brzyski RG, Diamond MP, Coutifaris C, Schlaff WD, Casson $P$, Christman GM, Huang $H$, Yan $Q$, Alvero R, Haisenleder DJ. Letrozole versus clomiphene for infertility in the polycystic ovary syndrome. N Engl J Med. 2014 Jul 10; 371: 119-29.

12. Arentz S, Abbott JA, Smith CA, Bensoussan A. Herbal medicine for the management of polycystic ovary syndrome (PCOS) and associated oligo/amenorrhoea and hyperandrogenism; a review of the laboratory evidence for effects with corroborative clinical findings. BMC complementary and alternative medicine. 2014 Dec; 14(1): 1-9.

13. Sathyapalan T, David R, Gooderham NJ, Atkin SL. Increased expression of circulating miRNA-93 in women with polycystic ovary syndrome may represent a novel, noninvasive biomarker for diagnosis. Scientific reports. 2015 Nov 19; 5(1): 1-8.

14. McCartney CR, Marshall JC. Polycystic ovary syndrome. New England Journal of Medicine. 2016 Jul 7;375(1): 54-64. 
15. Genazzani AD. Inositol as putative integrative treatment for PCOS. Reproductive biomedicine online. 2016 Dec 1; 33(6): 770-80.

16. Qi X, Pang Y, Qiao J. The role of anti-Müllerian hormone in the pathogenesis and pathophysiological characteristics of polycystic ovary syndrome. European Journal of Obstetrics \& Gynecology and Reproductive Biology. 2016 Apr 1; 199: 82-7.

17. Szydlarska D, Machaj M, Jakimiuk A. History of discovery of polycystic ovary syndrome. Advances in Clinical and Experimental Medicine. 2017 May 1; 26(3): 555-8.

18. Macut D, Bjekić-Macut J, Rahelić D, Doknić M. Insulin and the polycystic ovary syndrome. Diabetes research and clinical practice. 2017 Aug 1; 130: 163-70.

19. Chen S, Jiang M, Ding T, Wang J, Long P. Calprotectin is a potential prognostic marker for polycystic ovary syndrome. Annals of clinical biochemistry. 2017 Mar; 54(2): 253-7.

20. Karakas SE. New biomarkers for diagnosis and management of polycystic ovary syndrome. Clinica Chimica Acta. 2017 Aug 1; 471: 248-53.

21. Neven AC, Laven J, Teede HJ, Boyle JA. A summary on polycystic ovary syndrome: diagnostic criteria, prevalence, clinical manifestations, and management according to the latest international guidelines. In Seminars in reproductive medicine 2018 Jan (Vol. 36, No. 01, pp. 005-012). Thieme Medical Publishers.

22. Jin P, Xie Y. Treatment strategies for women with polycystic ovary syndrome. Gynecological Endocrinology. 2018 Apr 3; 34(4): $272-7$
23. Wolf WM, Wattick RA, Kinkade ON, Olfert MD. The current description and future need for multidisciplinary PCOS clinics. Journal of clinical medicine. 2018 Nov; 7(11): 395.

24. Raperport C, Homburg R. The source of polycystic ovarian syndrome. Clinical Medicine Insights: Reproductive Health. 2019 Aug; 13: 1179558119871467.

25. Rao P, Bhide P. Controversies in the diagnosis of polycystic ovary syndrome. Therapeutic Advances in Reproductive Health. 2020 Jun; 14: 2633494120913032.

26. Joham AE, Kakoly NS, Teede HJ, Earnest A. Incidence and Predictors of Hypertension in a Cohort of Australian Women With and Without Polycystic Ovary Syndrome. The Journal of Clinical Endocrinology \& Metabolism. 2021 Jun; 106(6): 1585-93.

27. Lin AW, Siscovick D, Sternfeld B, Schreiner P, Lewis CE, Wang ET, Merkin SS, Wellons M, Steffen L, CalderonMargalit R, Cassano PA. Associations of diet, physical activity and polycystic ovary syndrome in the Coronary Artery Risk Development in Young Adults Women's Study. BMC Public Health. 2021 Dec; 21(1): 1-0.

28. Tabassum F, Jyoti C, Sinha HH, Dhar K, Akhtar MS. Impact of polycystic ovary syndrome on quality of life of women in correlation to age, basal metabolic index, education and marriage. Plos one. 2021 Mar 10; 16(3): e0247486.

29. Bruni V, Capozzi A, Lello S. The Role of Genetics, Epigenetics and Lifestyle in Polycystic Ovary Syndrome Development: the State of the Art. Reproductive Sciences. 2021 Mar 11; $1-2$.

Source of Support: The author(s) received no financial support for the research, authorship, and/or publication of this article.

Conflict of Interest: The author(s) declared no potential conflicts of interest with respect to the research, authorship, and/or publication of this article.

For any question relates to this article, please reach us at: editor@globalresearchonline.net New manuscripts for publication can be submitted at: submit@globalresearchonline.net and submit_ijpsrr@rediffmail.com 